\title{
WORK AND HEALTH IN TRANSITION: TRENDS OF SUBJECTIFICATION IN APPLIED PSYCHOLOGY
}

\author{
Severin Hornung ${ }^{1}$, Matthias Weigl ${ }^{2}$, Britta Herbig ${ }^{3}$, \& Jürgen Glaser ${ }^{4}$ \\ ${ }^{1}$ Institute of Psychology, University of Innsbruck (Austria) \\ ${ }^{2}$ Institute and Outpatient Clinic for Occupational, Social and Environmental Medicine, LMU University \\ of Munich (Germany) and Institute for Patient Safety (IfPS), University Hospital Bonn (Germany) \\ ${ }^{3}$ Institute and Outpatient Clinic for Occupational, Social and Environmental Medicine, \\ LMU University of Munich (Germany) \\ ${ }^{4}$ Institute of Psychology, University of Innsbruck (Austria)
}

\begin{abstract}
Reported is the synthesis of a series of seven studies on work and health, conducted collaboratively by researchers in applied psychology and occupational medicine. This qualitative meta-study develops a framework, in which reviewed studies are structured, aggregated, integrated, and interpreted in a theory-guided iterative process of themed analysis. Building on empirical results, the subsequent interpretive integration seeks to demonstrate, how overarching, pervasive, and in psychological research typically underemphasized tendencies of "subjectification" manifest in exemplary work contexts, research topics, and results. Subjectification of work is operationalized in dimensions of work intensification (performance focus), work internalization (goal adoption), and work individualization (job personalization). A meta-dimension is work insecurity (personal risk), cultivated in contemporary management ideologies of employee self-reliance. Following thematic description, content-analytical structuring criteria include: a) focus on work task (activity) versus working conditions (context); b) primary (close, direct, explicit) versus secondary (inferred, indirect, subtle) references to and/or indication for identified tendencies of subjectification; and c) theoretically assumed and empirically examined relationships with negative (psychopathological) and positive (psychosalutogenic) short, medium, and longer-term attitudinal and health-related work effects, as well as the personality-shaping impact of long-term occupational socialization. Psychological aspects of work tasks are core to 4 studies, 3 focus on working conditions and organizational practices. References to intensification were dominant in 4 studies, whereas 5 include internalization processes, and 3 predominantly focus on individualization of work. All studies share secondary or indirect references to other subjectifying tendencies. Examined work effects were aggregated into a matrix of short, medium and long-term positive and negative manifestations of health and wellbeing. Results suggest tensions and pressures arising from the motivational individualization of work tasks and conditions, resulting internalization of organizational interests and goals (e.g., performance, efficiency, costs), coupled with system-inherent tendencies of work intensification. These dysfunctional dynamics constitute risks factors for psychologically detrimental or harmful forms of self-management, self-control, and self-endangering work behavior, as manifestations of "internalized" incompatibilities between work and health in the neoliberal workplace, aggravated by existential threats associated with political-economic crisis. Outlined are implications of subjectification for a critical reevaluation and reorientation of basic theoretical assumptions of research and practice in applied psychology and occupational health.
\end{abstract}

Keywords: Work and health, neoliberal transformation, work systems analysis, subjectification of work.

\section{Introduction}

Fundamental transitions in the world of work require overarching frameworks of challenges facing applied psychology and related medical and social science disciplines. Public, occupational, and individual health and wellbeing need to be understood within broader developments in society (Bliese, Edwards, \& Sonnentag, 2017; Hornung \& Höge, 2019; Korunka \& Kubicek, 2017). Contributing to a reflexive, interdisciplinary, and humanization-oriented perspective, this article presents a conceptually grounded meta-study of seven empirical investigations. Part of an interdisciplinary collaboration with researchers in occupational medicine, studies address health-relevant aspects of work activities in 
contemporary organizations. The synthesis aims to show, how these topics are, on a meta-level, interconnected and interdependent. This entails identifying and analyzing major trends in the transition of work and assessing their relevance for the relationships between work and health, characterized by conflicts, synergies, and trade-offs, as well as exploring, how these tendencies are reflected in topics, constructs, and results of the reviewed study portfolio. The methodological approach of this qualitative meta-study is informed by themed analysis, an iterative content-analytical process of theory-based and exploratory (deductive-inductive) categorization, structuring, and synthesis (Munro, 2012). Derived from the interdisciplinary literature and suggested as dominant tendencies in transitional patterns of work and employment in advanced neoliberal economies, are tendencies of intensification, internalization and individualization. These interdependent yet distinct trends are exemplified by embedding reviewed studies from applied psychology and occupational medicine into the broader socio-cultural and historical dynamics of societal, economic, and workplace change. A field of tensions among intensification, internalization, and individualization is suggested as a framework work in transition. This approach reconciles research in psychology with analyses in social science disciplines, such as history, anthropology, sociology, and critical management studies, on the "subjectification" of work (Becke, 2017), "biopower" and "neoliberal governmentality" (Munro, 2012) in post-disciplinary work systems (Weiskopf \& Loacker, 2006). Against this background, this review is oriented towards determining, how tendencies of subjectivation, specifically, dynamics of work intensification (escalating performance requirement), internalization (employee identification and goal adoption), and individualization (personalization of work tasks and conditions), are reflected in examined research topics and results. Following thematic description and classification, integrating theories and assumptions of work psychology, three main content-analytical structuring schemes were applied: a) focus on work task (activity) versus working conditions (context); b) primary (close, direct, explicit) versus secondary (inferred, indirect, subtle) references to and/or indication for identified tendencies of subjectification; and c) theoretical and empirical relationships with positive and negative short, medium, and long-term motivational, health and socializing (personality-shaping) work effects.

\section{Transformation of work as subjectification}

Thematic analysis involved extracting trends from the psychological, sociological, economic, and management literature on the transformation of employment, work, and organizations in the post-industrial (also post-Fordist, post-Taylorist, post-modern, post-disciplinary) era of advanced neoliberal capitalism as dominating (hegemonic) political-economic regime. This review generated major themes associated with the "subjectification of work" (Becke, 2017; Pongratz \& Voss, 2003; Weiskopf \& Loacker, 2006), sorted into three dimensions: a) intensification; b) internalization; c) individualization. A meta-dimension, energizing tendencies of subjectification, is work insecurity (personal risk), associated with increasing precariousness in employment practices, cultivated in neoliberal management ideologies of employee self-reliance. Theoretically generalizable and empirically established consequence of economic primacy (profit maximization, cost reduction), work intensification refers to performance "improvements" by increasing the (quantitative) amount of work and/or other (qualitative) requirements (Burchell, Ladipo \& Wilkinson, 2002; Korunka \& Kubicek, 2017). Temporal extension (extensification) of work, is also subsumed under intensification here (Allvin, Aronsson, Hagström, Johansson, \& Lundberg, 2011). For "self-managing" employees, intensification manifests as tensions between positive challenges and occupational learning opportunities versus work overload and stressors, including "externalized" (long-term) health (care) costs in precarious employment arrangements. Focal in psychology, processes of internalization refer to employee adoption and integration of organizational (managerial, performance-oriented) norms, goals, and values (Brown, 2017). Tensions manifest as ambiguities between motivation and self-regulation at work versus "self-executed" work stressors and motivated self-endangerment, threatening erosion of long-term health and work ability, reinforced by indirect (subjectifying) organizational (managerial) control practices, e.g., delegation of responsibility, goal setting, and self-management (Glaser, Hornung, \& Höge, 2019). Work individualization refers to de-standardization, de-formalization, flexibilization and personalization of working conditions, downsides of which are erosion of employment standards, collective agreements, and other protective labor political instruments (Allvin et al., 2011). For employees, this opens up conflictual areas of tension between self-determination and self-design of work tasks versus insecurity, social isolation and coping behavior for self-responsible fulfillment of expansive and/or unpredictable performance and behavioral demands. Increasing importance of such tensions, contradictions and paradoxes has been identified as a central characteristic of (post-)modern and post-disciplinary high-performance work systems. 


\section{Psychology of work and health}

The second thematic frame relates to the core of work psychology, the human-centered analysis, evaluation, and design of work systems and processes (Oesterreich and Volpert, 1986). In the Organization-Task/Activity-Individual (OTI) approach (Büssing, 1992), work activity is the nexus of interdependent and reciprocal influences between organizational structure (division of labor, supervision, discretion) and working individual (occupational health, skills, attitudes). Although work activity, individual, and organization appear to correspond with intensification, internalization, and individualization, likely, these trends are affecting all three domains. The following section provides some background theorizing on work systems and health impacts of work. A systems-theory perspective posits dialectical relationships between work system structure and dynamics (antagonisms, tensions, interdependencies, emergent properties), both within and across levels of analysis (Glaser et al., 2019). Organizational-level work system (structure) and work process (dynamic) partly determine individual-level job features, partitioned into work tasks (activity) and working conditions (context). The "primacy of the work task", puts the focus of action regulation on the work activity, distinguished from the conditions, situation, or context these actions are performed in (Büssing, 1992). The work task-conditions (activity-context) separation bears similarities with intrinsic (task-inherent) versus extrinsic (situation-related) job characteristics, but carries activity- and systems-theoretical assumptions on dynamic interactions between work activity, acting individual, and socio-technical embedding in organizational structures (Weber \& Jeppesen, 2017). This distinction served as initial structuring element, based on the possibly differential impact of identified transitions on the structure and dynamics of contemporary work systems. Intersection of work psychology and occupational medicine, health-effects were divided into psychopathological health impairments and psychosalutogenic (motivational, health-preserving, personality-promoting) aspects (Huppert, 2009; Stansfeld \& Candy, 2006). Categorization of content and temporal horizon yields a matrix of a) positive and b) negative manifestations, in the: i) short to medium-term, ii) medium to long-term, and iii) longer-term perspective (Frese, 1982). In the latter, relatively stable individual predispositions are assumed to change over longer periods of occupational socialization.

\section{Methods}

Synthesized are 7 empirical studies (S1-S7), conducted at university institutes of occupational medicine and psychology in Germany and Austria, presented between 2015-2019 at the Annual Scientific Congress of the German Association of Environmental and Occupational Medicine (55th-59th DGAUM). Most studies are cross-sectional. The exception, S4 reports data from a long-term longitudinal study with 4 measurement points on working conditions of resident hospital physicians. The occupational context of S2 is geriatric care, analyzing a statewide representative sample of elderly care homes. Studies S6 and S7 use heterogeneous convenience samples. S1 and S3 are in the German public administration; S5 in a Chinese telecommunications company. All constructs are measured with multi-item self-report questionnaires, assessed for validity and reliability (exploratory and/or confirmatory factor analysis, Cronbach's alpha, scale modifications). Correlational hypotheses are mostly tested in path or structural equation models (S1-S5). In two cases, multiple moderated linear regressions involved testing of interaction effects (S6, S7). Modeling approaches range from manifest-variable (scale-level) path model (S1) to complete (item-level) latent-variable structural equation models (S5), including hybrid combination (S2), and item parceling techniques (S2, S3, S4). One of these studies also tests interaction effects (S3). One uses a complex longitudinal design, with latent variables aggregating constructs across measurement points (S4). All data were verified; some analyses extended; arguments and theorizing integrated. Some construct labels were adapted for precision or emphasizing parallels across studies (e.g., identification vs. commitment). Themed analysis involved an iterative process of theory-guided (deductive) categorizations and explorative (inductive) analyses to identify recurring themes. Studies were structured according to chronological order, similarities in theories, constructs, methods, samples, and outcomes. Brief summaries and short titles were developed as content-descriptors. Three structuring schemes were applied: a) Work system focus distinguishes between the central role of the work task (activity) versus working conditions (context); b) Subjectification of work operationalizes primary (direct, explicit) and secondary (indirect, implicit) connections with tendencies of intensification, internalization, and individualization of work; c) Work-health impact integrates theoretical and empirical relationships with positive and negative short, medium, and long-term motivational, health-related and personality-shaping socializing work effects. Structured by these categories, synthesis results are reported next. 


\section{Results}

Studies share survey methodology, correlational analysis, occupational health focus, overlapping theories, and constructs. Short titles reflect core topics: S1-Work characteristics; S2-Interaction work; S3-Management practices; S4-Work extensification; S5-Job crafting; S6-Work self-redesign; S7-Negotiated fairness. Differentiating the structure (dynamics) of the work system (work process) into work task (work activity) and working conditions (execution context), focal trends were analyzed in both domains. Accordingly, 4 studies refer directly to work tasks and psychological regulation (S1, S2, S5, S6); 3 are more distal to the work activity, focusing on "extrinsic" working conditions as the context of action regulation (S3, S4, S7). Trends of intensification, internalization, individualization were analyzed in both groups, considering different perspectives. Direct references to intensification made 4 studies (S1-S4); 5 studies explicitly examine internalization processes (S1-S3, S5, S6); 3 focus on individualization of work organizations (S5-S7). All share secondary references to other subjectivation tendencies. Core studies focusing on the work activity are the investigation of positive and negative effects of work characteristics according to the job demands-resources model in S1, and the modeling of emotional regulation and health in interactive work in human services in S2. Both have a focus on intensification (time pressure, excessive demands) and internalization processes (organizational and professional identification). Two more studies relate to work tasks (S5, S6), investigating psychological internalization (work motivation, identification, meaning) from an individualization perspective. Focal in S5 are antecedents and motivational effects (psychological empowerment) of self-regulation and task modification. A follow-up, S6 examines proactive self-design of work and implications for quality of working life, focusing on interactive effects of task-related modes of control. The other 3 studies focus on working conditions (context), emphasizing intensification, internalization, individualization to different degrees. Investigating ambiguous effects of employee-oriented management practices on work ability, S3 supports internalization and intensification perspectives. In S4, the focus was on intensification (or extensification) with long-term effects of role conflicts between work and family on affective disorders (depression, anxiety). Part-time work introduced individualization aspect, work priority an internalization component. Individualization was central in S7, examining fairness perceptions regarding personalized working conditions, also including intensification and internalization processes (individual negotiation as management practice). Positive (psycho-) salutogenic effects on wellbeing and health are associated with psychological identification and internalization, specifically, opportunities for need-based personalization of work arrangements. Short to medium term, this refers to positive affect, intrinsic work motivation, and psychological empowerment (S1, S5); medium to long term to organizational identification (S1, S3, S6), self-efficacy, meaning, justice perceptions (S6, S7), and maintaining work ability (S3). Socialization processes apply to personality constructs (included as independent variables) of autonomy orientation and individual growth need (S1, S5), interaction competence, and functional emotion regulation (S2). Psychopathological work effects impairing well-being and health are associated with intensification. This includes short-term negative emotions (S2) and medium-term psychological irritation (S1). Prototypical longer-term effects are burnout (S2), psychosomatic complaints, and affective disorders (S1, S4, S6). Long-term negative socialization is captured in learned helplessness and alienation (S1) dysfunctional emotional regulation (S2) and trait components of depression and anxiety (S4).

\section{Discussion}

This meta-study illustrates tendencies of intensification, individualization and internalization in applied psychological research. A series of studies was structured and interpreted in a deductive-inductive synthesis. Identified tensions include ambiguous implications of motivational work design and personalization of work tasks or conditions, when employees internalize expansive and intrusive organizational (occupational, social) demands and norms, specifically, health-damaging effects of self-directed work intensification (Burchell et al., 2002; Hornung \& Höge, 2019; Korunka \& Kubicek, 2017). Associations between internalization of organizational (performance-related) goals and behavior-based (self-directed) intensification correspond with the subjectification thesis in work sociology, stressing relevance of self-endangering work behavior as harmful, interest-based "self-exploitation", facilitated through manipulation and indirect control (e.g., goal setting) in the context of job and income insecurity, as well as "externalization" of social costs and risks into the individual sphere of responsibility (Pongratz, \& Voß, 2003; Weiskopf \& Loacker, 2006). Tendencies of subjectification are conceived not as independent, but interacting "synergistically" towards observed psychological impacts. Tension among subjectifying tendencies were analyzed on a conceptual basis and study results explored for indications. The dialectics between intensification and internalization are arguably most central and paradox, relating to conflict and convergence of interests in employment. Work 
intensification in the form of externally mandated increases in performance demands trigger resistance and run counterproductive to employee identification with the organization. Internalized (subjectified) work intensification and flexibility are exercised in an "organic" way, bypassing protective mechanisms of employee reactance (Hornung \& Höge, 2019). This highlights tensions between motivational personalization of work, employee identification with (inherently expansive) organizational performance requirements, and health-damaging effects of resulting self-enacted work stressors. To capture the dynamic complex interrelationships between work and health, applied psychology and occupational medicine need to revise basic assumptions on the motivational basis of work behavior, considering research in the social sciences on subjectification and governmentality in post-disciplinary work regimes. Increasingly relevant are mechanisms of indirect control through internalized performance norms and psychologically harmful self-management. This "subjectified intensification" of work coincides with erosion of institutional and social protective factors (e.g., employee rights, unions, solidarity) through neoliberal flexibilization and individualization. Work design needs to counteract these tendencies. Occupational psychology and medicine must become ethical instances of social responsibility, transcending disciplinary, organizational and administrative boundaries, actively standing in for the wellbeing of their main constituents.

\section{References}

Allvin, M., Aronsson, G., Hagström, T., Johansson, G., \& Lundberg, U. (2011). Work without boundaries: Psychological perspectives on the new working life. Chichester: Wiley.

Becke, G. (2017). The subjectivation of work and established-outsider figurations. Historical Social Research, 42, 93-113. doi:10.12759/hsr.42.2017.4.93-113

Bliese, P. D., Edwards, J. R., \& Sonnentag, S. (2017). Stress and well-being at work: A century of empirical trends reflecting theoretical and societal influences. Journal of Applied Psychology, 102, 389-402. doi:10.1037/ap10000109

Brown, A. D. (2017). Identity work and organizational identification. International Journal of Management Reviews, 19, 296-317. doi:10.1111/ijmr.12152

Burchell, B., Ladipo, D., \& Wilkinson, F. (Eds.). (2002). Job insecurity and work intensification. London: Routledge.

Büssing, A. (1992). Organisationsstruktur, Tätigkeit und Individuum [Organizational structure, activity, and individual]. Bern: Huber.

Frese, M. (1982). Occupational socialization and psychological development: An underemphasized research perspective in industrial psychology. Journal of Occupational Psychology, 55, 209-224. doi:10.1111/j.2044-8325.1982.tb00095.x

Glaser, J., Hornung, S., \& Höge, T. (2019). Organizational tensions, paradoxes, and contradictory demands in flexible work systems. Journal Psychologie des Alltagshandelns / Psychology of Everyday Activity, 12(2), 21-32.

Hornung, S., \& Höge, T. (2019). Humanization, rationalization or subjectification of work? Employee-oriented flexibility between i-deals and ideology in the neoliberal era. Business \& Management Studies: An International Journal, 7, 3090-3119. doi: 10.15295/bmij.v7i5.1384

Huppert, F. A. (2009). Psychological well-being: Evidence regarding its causes and consequences. Applied Psychology: Health and Well-Being, 1, 137-164. doi:10.1111/j.1758-0854.2009.01008.x

Korunka, C., \& Kubicek, B. (2017). Job demands in a changing world of work. Impact on workers' health and performance and implications for research and practice. Cham: Springer.

Munro, I. (2012). The management of circulations: Biopolitical variations after Foucault. International Journal of Management Reviews, 14, 345-362. doi:10.1111/j.1468-2370.2011.00320.x

Oesterreich, R., \& Volpert, W. (1986). Task analysis for work design on the basis of action regulation theory. Economic and Industrial Democracy, 7, 503-527. doi:10.1177/0143831X8674005

Pongratz, H. J., \& Voß, G. G. (2003). From employee to 'entreployee': Towards a 'self-entrepreneurial' work force? Concepts and Transformation, 8, 239- 254. doi:10.1075/cat.8.3.04pon

Stansfeld, S., \& Candy, B. (2006). Psychosocial work environment and mental health-a meta-analytic review. Scandinavian Journal of Work, Environment \& Health, 32, 443-462. doi:10.5271/sjweh. 1050

Weber, W. G. \& Jeppesen, H. J. (2017). Collective human agency in the context of organizational participation. Zeitschrift für Arbeits- und Organisationspsychologie, 61, 51-68. doi:10.1026/09324089/a000234

Weiskopf, R. \& Loacker, B. (2006). "A snake's coils are even more intricate than a mole's burrow." Individualization and subjectification in post-disciplinary regimes of work. Management Revue, 17, 395-419. doi:10.5771/0935-9915-2006-4-395 